\title{
Phasor Measurement Unit and Phasor Data Concentrator test with Real Time Digital Simulator
}

Diakos, Konstantinos; Wu, Qiuwei; Nielsen, Arne Hejde

Published in:

Proceedings of IEEE PES APPEEC 2014

Link to article, DOI:

10.1109/APPEEC.2014.7066096

Publication date:

2014

Link back to DTU Orbit

Citation (APA):

Diakos, K., Wu, Q., \& Nielsen, A. H. (2014). Phasor Measurement Unit and Phasor Data Concentrator test with Real Time Digital Simulator. In Proceedings of IEEE PES APPEEC 2014 IEEE.

https://doi.org/10.1109/APPEEC.2014.7066096

\section{General rights}

Copyright and moral rights for the publications made accessible in the public portal are retained by the authors and/or other copyright owners and it is a condition of accessing publications that users recognise and abide by the legal requirements associated with these rights.

- Users may download and print one copy of any publication from the public portal for the purpose of private study or research.

- You may not further distribute the material or use it for any profit-making activity or commercial gain

- You may freely distribute the URL identifying the publication in the public portal

If you believe that this document breaches copyright please contact us providing details, and we will remove access to the work immediately and investigate your claim 


\title{
Phasor Measurement Unit and Phasor Data Concentrator test with Real Time Digital Simulator
}

\author{
Konstantinos Diakos, Qiuwei Wu, Arne Hejde Nielsen \\ Centre for Electric Power and Energy \\ Department of Electrical Engineering \\ Technical University of Denmark \\ Kg. Lyngby, DK 2800, Denmark \\ Email: s121222@student.dtu.dk, qw@elektro.dtu.dk, ahn@elektro.dtu.dk
}

\begin{abstract}
The main focus of the electrical engineers nowadays, is to develop a smart grid that is able to monitor, evaluate and control the power system operation. The integration of Intelligent Electronic Devices (IEDs) to the power network, is a strong indication of the inclination to lead the power network to a more reliable, secure and economic operation. The implementation of these devices though, demands the warranty of a secure operation and high-accuracy performance. This paper describes the procedure of establishing a PMU (Phasor Measurement Unit)-PDC (Phasor Data Concentrator) platform that is able to derive and communicate synchrophasor measurements of different parts of the power network and the development of tests, according to IEEE standards, that evaluate the performance of PMUs and PDCs. The tests are created by using a Real Time Digital Simulation (RTDS) system. The results obtained from testing are analyzed to evaluate the performance of the devices.
\end{abstract}

Index Terms-- Phasor Measurement Unit testing, Phasor Data Concentrator testing, Real Time Digital Simulator, Smart Grid.

\section{INTRODUCTION}

As the need to create a smart power grid is increasing, it is of high importance to integrate Intelligent Electronic Devices (IEDs) to the power system that will enable visualization, control and communication of the power system's performance. At the core of this effort, is the development of Wide Area Measurement Systems (WAMS) and Wide Area Monitoring Protection and Control Systems (WAMPACS) that extract consistent and time-accurate measurements from the power system in order to ensure its stable, secure and economic operation.

Fundamental components of the WAMS and WAMPACS are Phasor Measurement Units (PMUs) and Phasor Data Concentrators (PDCs). PMUs are devices able to sample high speed, time-stamped snapshots of voltage and current in phasor format, frequency and rate of change of frequency. Being synchronized to Coordinated Universal Time (UTC) through Global Positioning System (GPS) signal, these devices are able to provide real time measurements recorded from different parts of the power system, giving this way the potential to time-align these measurements and have a precise and representative view of the power system. PDCs are devices able to concentrate measurements from different PMUs, time-align them and communicate them as a single stream to other PDCs or to monitoring and control devices. Using the afore-mentioned devices, a synchrophasor network can be developed which can derive measurements from different parts of the power network through PMUs. These measurements can be aggregated and time-aligned through PDCs and sent as a single stream of data through the communication network to Supervisory Control and Data Acquisition systems (SCADA) or Energy Management Systems (EMS).

In order to ensure the reliable operation of PMUs and PDCs, protocols have been developed to set standards for the requirements of these devices. Thus, the next step is to evaluate the performance of PMUs and PDCs in consonance with the IEEE standards [1], [2], [3]. The aim of this paper is the development of a PMU-PDC platform with Real Time Digital Simulator (RTDS) that tests the performance requirements of PMUs and PDCs according to the IEEE standards. The following sections present an overview of the software and hardware employed for the platform, the use of the platform to conduct PMU and PDC tests, how the tests are created in RSCAD software of RTDS and a short result summary. The paper ends with the conclusion section.

\section{HARDWARE AND SOFTWARE EMPLOYED AT THE PLATFORM}

Further on, the hardware and software employed to create the PMU-PDC platform with RTDS is presented and the platform is shown in figure 1 .

\section{A. Real Time Digital Simulator}

Real Time Digital Simulator [4] simulates the operation of electrical power systems designed at the RSCAD interface software of the system and can also be used as a testing system for protection and control devices. The system also gives the ability to visualize the simulation results through monitoring applications of the RSCAD software. RTDS is 


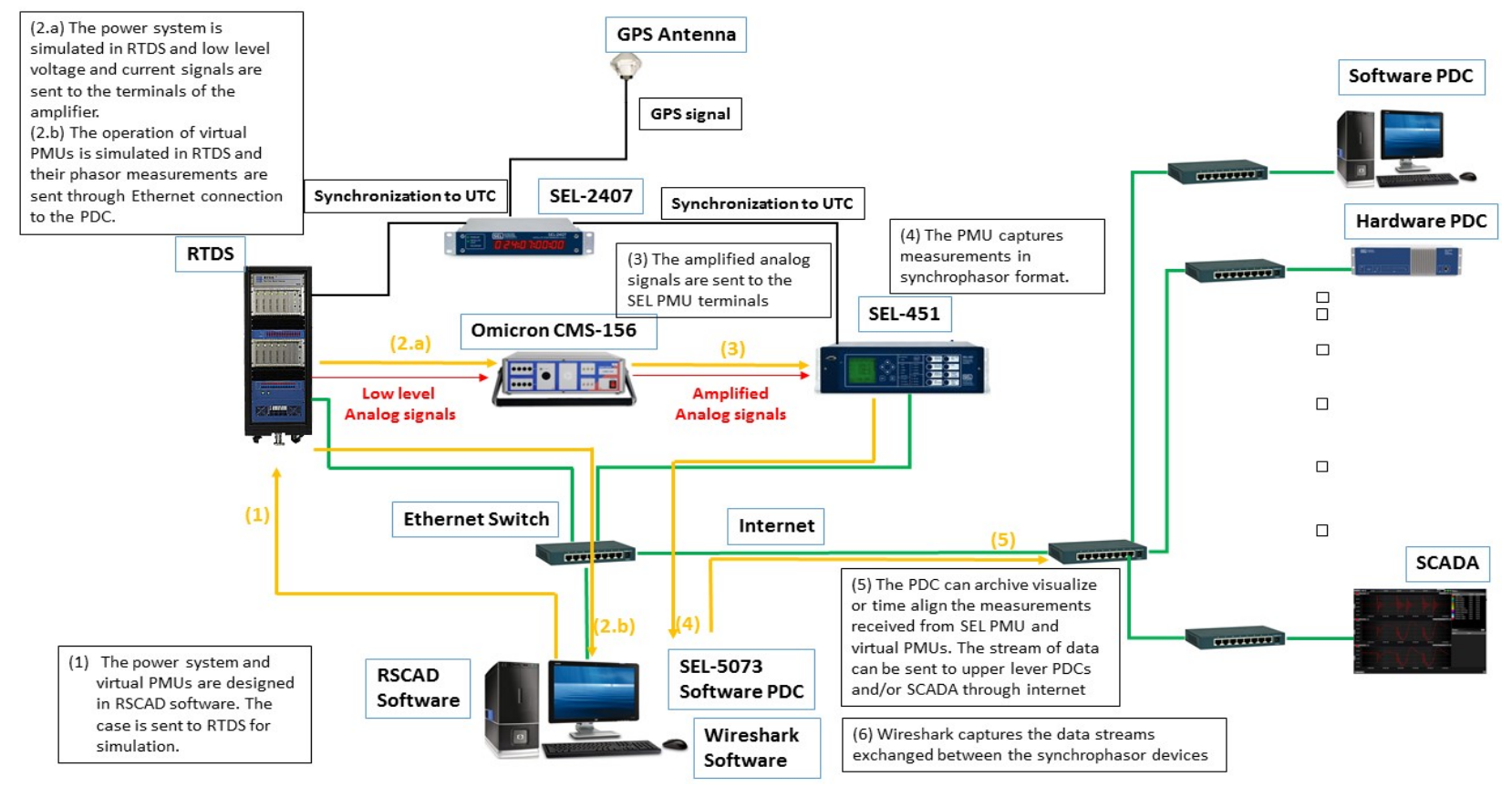

Figure 1. PMU-PDC test platform with RTDS

processing in parallel the data with PB5 or GPC processor cards mounted in racks. Each rack, besides processors, contains analog and digital input and output channels that enable the communication of RTDS with external devices. The RTDS system of the DTU LAB consists of ten racks. The first five racks consist of one PB5 and four GPC processor cards and the last five racks consist of four PB5 processor cards. Each processor card consists of two processors (A and B). A Giga-Transceiver Front Panel Interface (GTWIF) card for interaction with the RSCAD software is established for every rack. For the first four racks there are two GigaTransceiver Communication (GTNET) cards, one provides a real time communication link to and from the simulator via Ethernet and the other is a GTNET-PMU which provides communication with PMUs and is necessary for creating PMU applications in RTDS. There is an IRC switch which enables inter-rack communication and simultaneous use of them for large scale systems simulation. The racks also contain GigaTransceiver Digital Input (GTDI) and Giga-Transceiver Digital Output (GTDO) cards for receiving and transmitting digital signals to external devices (e.g. relays). There is a Giga-Transceiver Front Panel Interface (GTFPI) card that links the processors of the racks to a Low Voltage Digital Input/Output Interface Panel and a 250Vdc Digital Output Interface Panel. Each rack has Giga-Transceiver Analog Input (GTAI) and Giga-Transceiver Analog Output (GTAO) cards for importing and exporting analog signals to devices (e.g. PMUs). Finally the racks contain a GTSYNC card to synchronize the simulator through GPS signal to UTC.

\section{B. SEL-451 Phasor Measurement Unit}

The SEL-451 [5] device, is a distribution relay that besides protection operations can perform monitoring and data recording of high accuracy. As already mentioned it can be connected to several parts at the power network and because of the synchronization to UTC can capture high-speed, timestamped measurements of different parts of the power network. The configuration of the device is done with the use of the AcSELelerator Quickset software. SEL-451 can be serially connected through an RS-232 cable to a computer that has installed the AcSELerator Quickset software and the operation and communication settings of the device can be settled. The measurements that are captured by the device can be communicated to monitoring devices or to PDCs through Ethernet connection.

\section{Omicron CMS-156 Amplifier}

The Omicron CMS-156 [6] device is a three phase voltage and three phase current amplifier. Since the RTDS software produces low level analog signals from the simulated cases, these signals need to be amplified in order to feed the PMU terminals with actual signal values. Despite the fact that SEL451 has a special low level interface that can accept directly the low level signals of RTDS, that is not the case in the majority of PMUs. For this reason an amplifier is included to the platform to create a general procedure for PMU testing. The amplifier consists of three phase voltage with neutral and three phase current with neutral outputs that are galvanic isolated from the inputs. Three phase signals of up to 250 Volts and 25 Amperes can be produced and fed to the terminals of the PMU under test.

\section{SEL-2407 Satelite Synchronized Clock}

The SEL-2407 [7] device is a satellite synchronized clock that provides IRIG-B type time pulse output for the synchrophasor devices and the RTDS. Since there is need for having time aligned PMU measurements from different parts of the power network, the PMUs need to be synchronized to a 
common time-source. The time-source that is selected is the Coordinated Universal Time (UTC) and the PMUs and RTDS are synchronized to it through SEL-2407. The device is connected with a BNC cable to a GPS antenna that receives the UTC signal from satellites. The GPS signal is converted to IRIG-B type time pulse and is sent through BNC cables to the PMUs and the GTSYNC card of the RTDS. With UTC as time reference the actual PMUs and virtual PMUs (created in RTDS) can time stamp their measurements, thus enabling the PDC to time-align the received data and provide timeaccurate view of the power network operation.

\section{E. SEL-5073 Phasor Data Concentrator}

The SEL-5073 [8] is a software PDC that has to be installed to a computer to operate. The synchrophasor measurements captured by PMUs or other PDC can be aggregated by SEL-5073 PDC which further has the ability to time-align, archive and send as a single stream to other PDCs or perform calculations with these measurements. The exchange of data is done through Ethernet connection and the SEL-5073 supports the IEEE C37.118-2005 Standard for Synchrophasors for Power Systems. The software used to operate the SEL-5073 PDC is the PDC assistant. The operation of SEL-5073 at the PMU-PDC platform is to aggregate the data streams from the virtual PMUs and SEL451 , time-align the synchrophasor data, create a single data stream and send this data stream to a distant PDC.

\section{F. Wireshark}

Wireshark [9] is a network packet analyzer software that has the ability to capture and parse packets that are exchanged from IP to IP at an established network. At an established network, each device is assigned an IP address and every communication between the devices is accomplished with exchange of data at these IP addresses. The communication between the PMU, the PDC and the RTDS at the platform takes place through an Ethernet network. If Wireshark is operating while the PMU and PDC tests are in progress, the software is able to capture and visualize the packets of data that are exchanged between the devices. Wireshark can distinguish between data transfer protocols (TCP or UDP) and supports several communication protocols, among them is the C37.118 for exchange of synchrophasor measurements. Thus, by using this software besides the option of capturing data the user has the ability to see the measurements captured in a message format defined by IEEE Std C37.118.2-2011 [10].

\section{USE OF THE PLATFORM TO CREATE PMU AND PDC TESTS}

\section{A. PMU Testing}

As depicted in Fig. 1 voltage and current signals are generated in RSCAD software that simulate the steady and dynamic state condition of a power network. For the steady state, test cases that examine the PMU compliance with frequency range, signal magnitude range (voltage and current), phase angle range, harmonic distortion and out of band interference are created. For the dynamic state, test cases that examine the PMU compliance with modulation frequency of magnitude and phase, measurement of bandwidth, ramp of the frequency, step change in magnitude and phase and measurement reporting latency are created. The test cases are sent through Ethernet from RSCAD to RTDS where they are simulated in order to have an accurate and reliable representation of the power system operation in real time. At the RTDS low level analog output signals are produced which cannot be captured by the terminals of the PMU. Thus, the analog output signals are amplified by Omicron CMS-156 and sent to the terminals of the SEL-451 PMU. The PMU captures the voltage and current signals and creates measurements in synchrophasor format. The synchrophasor measurements of the PMU are sent back to the RTDS system, where they are analyzed for their accuracy and compliance to the IEEE standards

\section{B. PDC Testing}

In order to create test cases for the PDC device, virtual PMU components are imported in RSCAD to simulate the operation of real PMUs additionally to measurements captured by SEL-451. The operation of virtual PMUs is simulated in RTDS and measurements are sent through Ethernet connection to the SEL PDC. The SEL PDC also receives measurements from SEL-451 as it is described in the previous paragraph and has the ability to either archive, forward, or time-align the measurements that receives from different PMUs. The communication of data among the virtual PMUs, the SEL-451 PMU and the SEL-5073 PDC is captured by Wireshark software to test if the PDC meets the requirements described in [3]. The PDC tests performed through the synchrophasor platform are developed according to the requirements for PDC devices described in paragraph 7.4 of [3].

\section{DEVELOPMENT OF PMU AND PDC TESTS IN RSCAD}

\section{A. PMU tests in RSCAD}

In figure 2 the Draft case for the PMU tests is presented. The main idea of the case is to create voltage and current signals that simulate the operation of a power network and with the help of Runtime to modulate the magnitude, the frequency and the phase of the signals and introduce harmonic distortion or out of bound interfering signals according to IEEE standards. The voltage and current digital signals are created with the "PMU Test Waveform Control Panel" component of the RTDS library, which creates three phase Line-to-Line voltages and three phase Line-to-Neutral currents. The signals are directly sent to the terminals of a virtual PMU which is simulated in RSCAD with the GTNETPMU component. The GTNET-PMU component has the ability to simulate ideally the operation of up to eight PMUs, thus high accuracy time-stamped measurements captured from the signals, generated by the "PMU Test Waveform 
Control Panel", are created. In addition, the signals are transformed to Line-to-Neutral for voltage and amplified for current and are sent to the Digital to Analog component of RSCAD to be converted to analog form. The conversion of signals from digital to analog is taking place to the first processor of the first rack of RTDS and the signals are output at the terminals of the GTAO card of the first rack. The signals produced are low level, so they are amplified by Omicron CMS-156 and then sent to the terminals of the real PMU (SEL-451). The measurements captured from SEL-451 are sent back via Ethernet to RTDS and comparison between the measurements of the virtual and actual PMU and monitoring of the results is done at the Runtime interface of RSCAD.

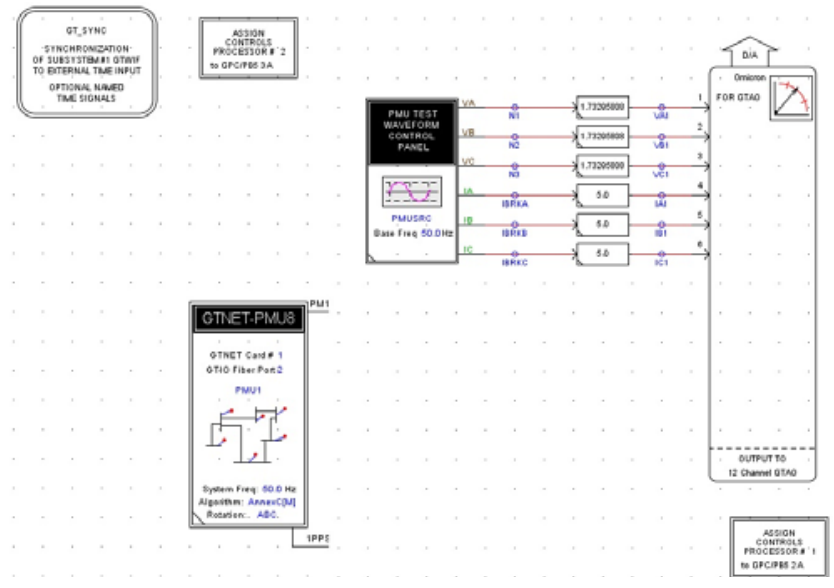

Figure 2. Draft case of the PMU tests

At the Runtime case of RSCAD push buttons enable the start and stop of the simulation, sliders modulate the respective values that are assigned in order to create the tests described in [1] and meters are monitoring the values that they are assigned during the test. To handle the modulation of the values in the Runtime, the RSCAD software has developed the "PMU Utility" tool [11] which is an interface designed create and run tests for the PMU performance. To run this tool, the draft case must contain a "PMU Waveform Control" component and a GTSYNC component. As described previously, the "PMU Waveform Control" component generates the test signals which are available at the GTAO card of RTDS. The connection to GTSYNC synchronizes the generated signals to 1 PPS. From the GTAO card outputs the signals are amplified and then sent to the terminals of the PMU under test. PMU utility communicates through Ethernet with the PMU under test, thus receiving the synchrophasor measurements generated and compares them with the theoretical measurements obtained by the virtual PMU of the Draft case.

\section{B. PDC tests in RSCAD}

In figure 3 the Draft case of the PDC tests is presented. The main idea of the PDC tests, is to create virtual PMUs that will capture synchrophasor measurements created by the "PMU Waveform Control" component, concurrently with
SEL-451, and send the data streams through Ethernet connection to the SEL-5073 software PDC. The PDC by its turn, aggregates the data streams from the different PMUs (actual and virtual), time-aligns the measurements and creates a single stream that is sent to another distant PDC. The exchange of data through the streams is captured by a network protocol analyzer program (Wireshark) which gives the ability to access the input and output data streams of the PDC and conclude about the performance. The need and purpose of using virtual PMUs, is that they can imitate perfectly the operation of actual PMUs and the communication and operation parameters of the virtual PMUs can be easily altered at the Draft case of RSCAD. In this way performance testing of PDCs can be achieved according to the requirements described in [3]. At the Draft case of RSCAD two controllers are created that intervene to the synchronization to UTC from the GTSYNC card and can simulate some of the time tests defined by the standards.

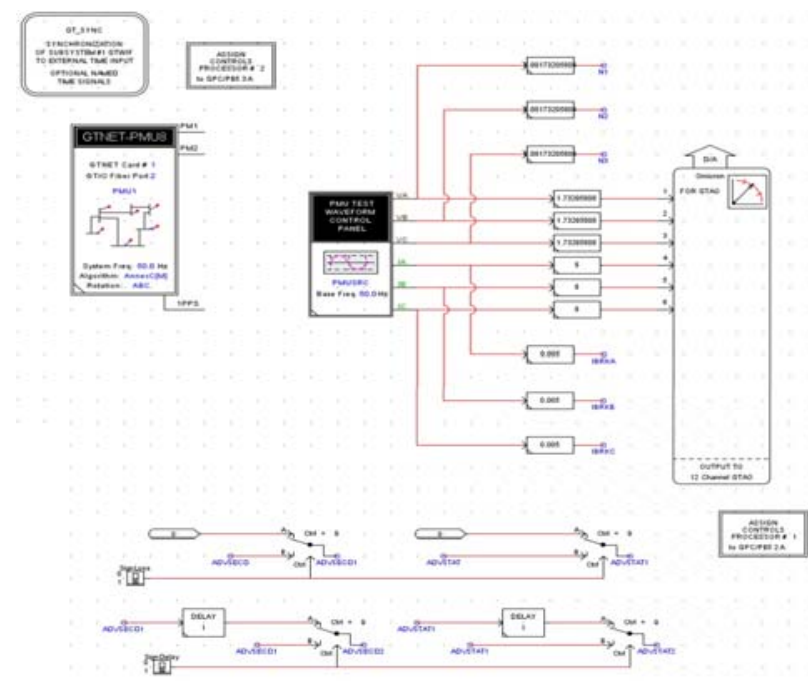

Figure 3. Draft case of the PDC tests

\section{TEST RESUlts}

\section{A. Results from PMU tests}

Using the PMU-PDC platform with RTDS the steady state and dynamic state tests defined in [1] are developed and performed for the SEL-451 PMU. For both test categories the synchrophasor measurements captured by the PMU under test are evaluated over the Total Vector Error (TVE), Frequency Error (FE) and Rate of Change of Frequency Error (RFE) as shown in the following equations. For the step change tests of dynamic state, the response time, delay time and maximum overshoot are also calculated. SEL-451 PMU conforms to the limits defined by the standards for both the steady and dynamic state tests. The tests are conducted at $50 \mathrm{~Hz}$ frequency and at 50 frames per second sampling rate for the PMU under test and are presented in table I and II. 
TABLE I. STEADY STATE TEST

\begin{tabular}{|c|c|c|c|c|c|c|c|c|}
\hline \multirow{2}{*}{\multicolumn{3}{|c|}{ Type of Test }} & \multicolumn{6}{|c|}{ Evaluation Quantities } \\
\hline & & & $\begin{array}{c}T V E \\
(\%)\end{array}$ & Limit & $F E$ & Limit & $R F E$ & Limit \\
\hline \multicolumn{3}{|c|}{ Frequency Range } & $\mathrm{V}$ & 1 & $\mathrm{~V}$ & 0.005 & $\mathrm{~V}$ & 0.01 \\
\hline \multicolumn{3}{|c|}{ Volate Magnitude } & V & 1 & $\vee$ & 0.005 & V & 0.01 \\
\hline \multicolumn{3}{|c|}{ Current Magnitude } & V & 1 & $\vee$ & 0.005 & V & 0.01 \\
\hline \multirow{2}{*}{$\begin{array}{l}\text { Phase } \\
\text { angle }\end{array}$} & \multicolumn{2}{|c|}{ Voltage } & V & 1 & $\mathrm{~V}$ & 0.005 & $\mathrm{~V}$ & 0.01 \\
\hline & \multicolumn{2}{|c|}{ Current } & $\mathrm{V}$ & 1 & $\mathrm{~V}$ & 0.005 & $\mathrm{~V}$ & 0.01 \\
\hline \multicolumn{3}{|c|}{$\begin{array}{l}\text { Harmonic } \\
\text { Distortion }\end{array}$} & V & 1 & V & 0.025 & V & 6 \\
\hline \multirow{6}{*}{$\begin{array}{c}\text { Out of } \\
\text { band }\end{array}$} & \multirow{2}{*}{$\begin{array}{c}46.6 \\
\mathrm{~Hz}\end{array}$} & $\mathrm{~V}$ & $\mathrm{~V}$ & 1.3 & $\mathrm{~V}$ & 0.01 & $\mathrm{X}$ & 0.1 \\
\hline & & $\mathrm{I}$ & $\mathrm{V}$ & 1.3 & $\mathrm{~V}$ & 0.01 & $\mathrm{X}$ & 0.1 \\
\hline & \multirow{2}{*}{$\begin{array}{l}50 \\
\mathrm{~Hz}\end{array}$} & $\mathrm{~V}$ & $\mathrm{~V}$ & 1.3 & $\mathrm{~V}$ & 0.01 & $\mathrm{X}$ & 0.1 \\
\hline & & I & V & 1.3 & $\mathrm{~V}$ & 0.01 & $\mathrm{X}$ & 0.1 \\
\hline & \multirow{2}{*}{$\begin{array}{c}53.3 \\
\mathrm{~Hz}\end{array}$} & $\mathrm{~V}$ & $\mathrm{~V}$ & 1.3 & $\mathrm{~V}$ & 0.01 & $\mathrm{X}$ & 0.1 \\
\hline & & I & $\mathrm{V}$ & 1.3 & $\mathrm{~V}$ & 0.01 & $\mathrm{X}$ & 0.1 \\
\hline
\end{tabular}

TABLE II. DYNAMIC STATE TESTS

\begin{tabular}{|c|c|c|c|c|c|c|c|c|}
\hline \multirow{2}{*}{\multicolumn{3}{|c|}{ Type of Test }} & \multicolumn{6}{|c|}{ Evaluation Quantities } \\
\hline & & & \multirow{2}{*}{$\begin{array}{c}T V E \\
(\%)\end{array}$} & \multirow{2}{*}{$\begin{array}{c}\text { Limit } \\
3 \\
\end{array}$} & \multirow{2}{*}{$\begin{array}{l}F E \\
\vee \\
\end{array}$} & \multirow{2}{*}{$\begin{array}{c}\text { Limit } \\
0.3\end{array}$} & \multirow{2}{*}{$\begin{array}{c}R F E \\
\vee \\
\end{array}$} & \multirow{2}{*}{$\begin{array}{c}\text { Limit } \\
30\end{array}$} \\
\hline \multirow{4}{*}{$\begin{array}{l}\text { Measu } \\
\text { rement } \\
\text { Band- } \\
\text { width }\end{array}$} & \multirow{2}{*}{$\begin{array}{l}\text { Phase and } \\
\text { Magnitude }\end{array}$} & $\mathrm{V}$ & & & & & & \\
\hline & & $\mathrm{I}$ & $\mathrm{V}$ & 3 & $V$ & 0.3 & $\mathrm{~V}$ & 30 \\
\hline & \multirow{2}{*}{ Phase only } & $\mathrm{V}$ & $\mathrm{V}$ & 3 & $V$ & 0.3 & V & 30 \\
\hline & & I & $\mathrm{V}$ & 3 & $\mathrm{~V}$ & 0.3 & $\mathrm{~V}$ & 30 \\
\hline \multirow{2}{*}{$\begin{array}{c}\text { Freque } \\
\text { ncy } \\
\text { Ramp }\end{array}$} & \multicolumn{2}{|l|}{ Voltage } & $\mathrm{V}$ & 1 & $\mathrm{X}$ & 0.005 & $\mathrm{X}$ & 0.1 \\
\hline & \multicolumn{2}{|l|}{ Current } & V & 1 & $\mathrm{X}$ & 0.005 & $\mathrm{X}$ & 0.1 \\
\hline \multicolumn{3}{|c|}{ Magnitue step change } & $\mathrm{V}$ & 1 & $\mathrm{~V}$ & 0.005 & $\mathrm{~V}$ & 0.1 \\
\hline
\end{tabular}

\section{B. Results from PDC tests}

From the requirements defined in [3] for the performance of PDC devices a number of tests is developed with the use of the platform. The ability of a PDC to pass these tests validates an accurate and reliable performance of the device. The PDC tests created by the platform and the results of testing the SEL5073 PDC are presented in table III and IV.

TABLE III. PDC TESTS

\begin{tabular}{|c|c|c|c|}
\hline \multirow{2}{*}{ Type of test } & \multicolumn{3}{|c|}{ Performance of SEL-5073 } \\
\hline & Subtest & Passed & Not Passed \\
\hline \multirow{4}{*}{ Time Test } & Loss of time signal & $\mathrm{V}$ & \\
\hline & UTC and Local Time & $\mathrm{V}$ & \\
\hline & Date and time change & $\mathrm{V}$ & \\
\hline & Timestamping Accuracy & $\mathrm{V}$ & \\
\hline \multirow{2}{*}{$\begin{array}{l}\text { Data } \\
\text { communication }\end{array}$} & TCP protocol & $\mathrm{V}$ & \\
\hline & UDP protocol & $\mathrm{V}$ & \\
\hline \multirow{5}{*}{ Configuration Test } & Configuration Frame & $\vee$ & \\
\hline & Interupt communication & $\mathrm{V}$ & \\
\hline & Change of PDC parameters & $\vee$ & \\
\hline & CFG frame request & $\vee$ & \\
\hline & Sanity Check & $\mathrm{V}$ & \\
\hline
\end{tabular}

TABLE IV. PDC TESTS

\begin{tabular}{|c|c|c|c|}
\hline \multirow{2}{*}{$\begin{array}{l}\text { Type of } \\
\text { test }\end{array}$} & \multicolumn{3}{|c|}{ Performance of SEL -5073} \\
\hline & Subtest & Passed & Not Passed \\
\hline \multirow{8}{*}{ Data Test } & Input data validation & $\mathrm{V}$ & \\
\hline & $\begin{array}{l}\text { Data aggregation with time } \\
\text { alignment }\end{array}$ & V & \\
\hline & $\begin{array}{l}\text { Data aggregation without time } \\
\text { alignment }\end{array}$ & & V \\
\hline & Data forwarding & $\vee$ & \\
\hline & $\begin{array}{l}\text { Data format and coordinate } \\
\text { conversion }\end{array}$ & V & \\
\hline & Phase and magnitude adjustment & $\mathrm{V}$ & \\
\hline & Reporting Rate Conversion & & $\mathrm{V}$ \\
\hline & Data latency & $\mathrm{V}$ & \\
\hline
\end{tabular}

\section{CONCLUSION}

The paper presents a PMU-PDC platform that is able to capture, aggregate, archive, time-align and distribute synchrophasor measurements from different parts of the power network and with the use of a RTDS system to create tests that evaluate the performance of PMU and PDC devices. By integrating a SEL-451 PMU, a SEL-5073 PDC, a SEL2407 satellite synchronized clock, a RTDS and an Omicron CMS-156 amplifier the platform is established and with the RSCAD software test cases to evaluate PMU and PDC performance are created. Finally, the SEL-451 PMU and the SEL-5073 PDC are tested using the created tests and the results of their performance are presented.

\section{REFERENCES}

[1] IEEE Standard for Synchrophasor Measurements for Power Systems, IEEE Std. C37.118.1 ${ }^{\text {TM }}$-2011, Dec 2011.

[2] IEEE Guide for Synchronization, Calibration, Testing and Installation of Phasor Measurement Units (PMUs) for Power System Protection and Control, IEEE Std. C37.242 ${ }^{\mathrm{TM}}-2013$, Mar 2013.

[3] IEEE Guide for Phasor Data Concentrator Requirements for Power System Protection, Control and Monitoring, IEEE Std. C37.244 ${ }^{\mathrm{TM}}$ 2013, Mar 2013.

[4] RTDS and RSCAD technical information [Online]. Available: http://www.rtds.com/index/index.html

[5] SEL-451 Relay Protection, Automation and Control System Instruction Manual, Schweitzer Engineering Laboratories, Inc., Pullman, WA, 2012.

[6] Omicron CMS-156 Reference Manual.

[7] SEL-2407 Satellite-Synchronized Clock Instruction Manual, Schweitzer Engineering Laboratories, Inc., Pullman, WA, 2012.

[8] SEL-5073 Synchrowave Phasor Data Concentrator Instruction Manual, Schweitzer Engineering Laboratories, Inc., 20130311.

[9] Wireshark User's Guide for Wireshark 1.10.

[10] IEEE Standard for Synchrophasor Data Transfer for Power Systems, IEEE Std. C37.118.2 ${ }^{\mathrm{TM}}$-2011, Dec 2011.

[11] PMU Utility User's Manual, RTDS Technologies, October 2013 rev. 1.0 . 